\title{
Distribution of a Human Brain Carboxypeptidase B Capable of Cleaving $\beta$-Amyloid Precursor Protein (APP) in Normal and Alzheimer's Diseased Brain
}

\author{
Kyoko Itoh $^{1}$ and Akira Matsumoto \\ ${ }^{1}$ Department of Pathology and ${ }^{2}$ Department of Radiation Biophysics \& Genetics, Kobe University School of Medicine, \\ 7-5-1, Kusunoki-cho, Chuo-ku, Kobe 650-0017
}

Received April 9, 2001; accepted May 28, 2001

\begin{abstract}
The processing of $\beta$-amyloid precursor protein (APP) is considered critical for understanding the pathogenesis of Alzheimer's disease (AD). To elucidate the significance of APP processing enzyme, we studied immunohistochemically the distribution of APP-processing protease (human carboxypeptidase $B$ : $\mathrm{HBCPB}$ ) in the normal control brains and $A D$ brains, using anti-C14 antibody which recognizes C-terminal 14 amino acids of HBCPB. In the control brains, intense and diffuse C14-immunoreactivity was observed in the cytoplasm of pyramidal neurons of the hippocampus.

Moderate immunoreactivity was found in the cortical and subcortical neurons. In AD brains, C14-immunoreactivity was markedly decreased in the brain regions examined except for the brain stem and cerebellum. However, HBCPB was shown to be colocalized with $\beta$-amyloid protein $(A \beta)$ in neuritic plaques. In addition, neuritic plaques included C14immunoreactive microglia/macrophages. Our present studies indicate that the expression of a novel APP-processing protease is impaired in $A D$ brains and may suggest the possible role of HBCPB in the pathogenesis of $A D$.
\end{abstract}

Key words: Alzheimer's disease, Carboxypeptidase B, $\beta$-amyloid protein, $\beta$-amyloid precursor protein, Immunohistochemistry

\section{Introduction}

Extracellular accumulation of fibrillar $\beta$-amyloid protein $(A \beta)$ in the cerebral cortices and cerebrovascular wall is a hallmark of Alzheimer's disease (AD) [7, 23]. Since A $\beta$ deposition is considered to play a crucial role in the pathogenesis of $\mathrm{AD}$, regulation of $\beta$-amyloid precursor protein (APP) processing has been extensively studied. The amyloid cores of the neuritic plaque consist mainly of the 40 to 42 amino acids $A \beta$, derived from the proteolytic processing of the APP [7, 24].

APP is a transmembrane glycoprotein processed through at least two different pathways, categorized as amyloidogenic and non-amyloidogenic ones $[12,28]$. In the non-amyloidogenic pathway, cleavage by $\alpha$-secretase between $A \beta$ sequence Lys ${ }^{16}$ and $\mathrm{Leu}^{17}$ of APP results in the production of an $8-\mathrm{kDa} \mathrm{C}$-terminal APP fragment that is considered to be the precursor of the $\mathrm{p} 3$ peptides. In the

Correspondence to: Kyoko Itoh, Department of Neurosciences, Case Western Reserve University, School of Medicine, Rm 658, 2109 Adelbert Rd, Cleveland, OH 44106-4975, USA. amyloidogenic pathway, proteolytic cleavage by $\beta$-secretase at the $\mathrm{N}$-terminus of the $\mathrm{A} \beta$ sequence yields a $10-\mathrm{kDa} \mathrm{C}$ terminal fragment containing the entire $A \beta$ sequence at its $\mathrm{N}$-terminus. Cleavage of the $8-\mathrm{kDa}$ or $10-\mathrm{kDa}$ C-terminal APP fragments by $\gamma$-secretase yields the p3 peptides or $\mathrm{A} \beta$, respectively [12]. The $\mathrm{C}$-terminus of the $\mathrm{A} \beta$ peptide is heterogeneous, ending at residue 40 or 42 , suggesting the existence of at least two different $\gamma$-secretases. It has been reported that distinct secretases, namely a cysteine protease and a serine protease, generate the C-termini of A $\beta 1-40$ and A $\beta 1-42$, respectively [2].

Recently, we purified, from normal human hippocampus, a novel serine protease (HBCPB) capable of cleaving natural APP $[9,10]$. Briefly, a 40-kDa proteolytic activity that degrades native brain APP in vitro was purified and characterized, and the molecular analysis disclosed that the activity was identified as a novel protease belonging to the carboxypeptidase B (HBCPB) family. Although this protease is homologous to plasma HBCPB synthesized in the liver, it has specific domains including the C-terminal 14 amino acid residues. Western blot analysis, cDNA cloning, and Northern blot analysis suggested a brain-specific ex- 
pression of this protease.

In the present study, we studied the localization of HBCPB in normal human brains, using rabbit polyclonal anti-C14 antibody that recognizes 14 amino acids at the C-terminus of HBCPB and compared the expression with that found in Alzheimer's disease. Although intense and diffuse C14-immunoreactivity was observed in the neuronal cytoplasm of the cerebral cortices in the control brains, C14immunoreactivity was markedly decreased in $\mathrm{AD}$ brains. Our observations in $\mathrm{AD}$ brains suggest that an impaired expression of HBCPB may be linked with excess $A \beta$ formation and deposition in senile plaques.

\section{Materials and Methods}

Brains from five patients without a history of neurological or psychiatric disorders (age 68-83 years old, mean 69 years old, two females and three males), and brains from six patients (age 70-87 years old, mean 80 years old, five females and one male) with a clinical and neuropathological confirmation of Alzheimer's disease were examined. On neuropathological examination, the control brains were free from any significant neuropathological changes, including senile plaques, neurofibrillary tangles and neuronal loss. The diagnosis of Alzheimer's disease was made according to CERAD criteria [14]. The brains were fixed in a $10 \%$ formalin solution for at least 1 week. Sections from human brains containing the frontal, orbital, parietal, temporal, and occipital cortices, amygdala, hippocampal complex, putamen, caudate nucleus, globus pallidus, thalamus, midbrain, pons, medulla and cerebellum were processed for histological stainings which included modified methenamine silver staining (Reusche stain) and immunohistochemistry.

\section{Preparation of polyclonal antibodies against the 40-kDa protease}

The anti-C14 antibody was raised in rabbits against the oligopeptide, NH2-SNPPVEKLLPLSLK-COOH, corresponding to the unique $\mathrm{C}$-terminal 14 amino acid residues of the $40-\mathrm{kDa}$ protease, HBCPB. The specificity of the antibody was checked by Western blot analysis using the total hippocampal homogenate from various brains. This antibody detected the 40 - and $30-\mathrm{kDa}$ bands and the result is relevant to the finding that the $30-\mathrm{kDa}$ protein is the mature form of this protease. No immunoreactive bands were detected in the human liver homogenates, suggesting that anti-C14 antibody specifically recognizes the brain species as previously described [9].

\section{Immunohistochemical procedures}

Immunohistochemistry was performed on $4 \mu \mathrm{m}$-thick paraffin sections, following the standard protocols of the avidin-biotin-complex (ABC) method.

For demonstrating C14-immunoreactivity, the sections were deparaffinized and immersed in $0.3 \%$ hydrogen peroxide in distilled water for $5 \mathrm{~min}$ to quench endogenous peroxidase activity. The sections were then placed into 0.01
$\mathrm{M}$ citrate buffer ( $\mathrm{pH}$ 6.0) and microwaved at $750 \mathrm{~W}$ for a total of $9 \mathrm{~min}$, with brief interruptions at 3 and 6 min to replace the evaporated volume with distilled water. The slides were then cooled down to room temperature in the citrate buffer and transferred to PBS. The sections were incubated overnight in a moist chamber at $4{ }^{\circ} \mathrm{C}$ with the rabbit anti-C14 antibody diluted 1:100 in PBS containing $1 \%$ bovine serum albumin. The reaction product was visualized with 3,3'-diaminobenzidine tetrahydrochloride. As controls, the primary antiserum was replaced by either normal serum or the antiserum absorbed by C-terminal 14-amino acids of HBCPB prior to application.

For confocal laser microscopy, all sections were microwaved, and incubated overnight at $4{ }^{\circ} \mathrm{C}$ with anti-C14 antibody (rabbit polyclonal, dilution 1:100) and each of the following primary antibodies: anti-neurofilament protein (mouse monoclonal, DAKO-NF, 2F11, DAKO, 1:100), anti$\beta$-amyloid (mouse monoclonal, 4G8, SENETEK, 1:100), anti-phosphorylated tau (mouse monoclonal, anti PHFTAU, AT8, Innogenetics, 1:100), anti-GFAP (mouse monoclonal, DAKO, 1:100) and anti-human macrophage, CD68 (mouse monoclonal, KP1, DAKO, 1:100). After additional washes, sections were incubated in the dark and at room temperature for $2 \mathrm{hr}$ with TRITC-conjugated goat anti-rabbit immunoglobulins at 1:100 dilution (for HBCPB immunofluorescence) and with FITC-conjugated goat anti-mouse immunoglobulins at 1:100 dilution (for $\mathrm{A} \beta$, phosphorylated tau, GFAP, CD68 and NF immunofluorescence). After the final washes, the sections were mounted and kept in the dark until analysis with a laser scanning confocal microscope (Confocal Imaging, Bio-Rad, USA).

\section{Results}

Light microscopy

Normal brain (Fig. 1A-E, Fig. 2A, B)

Variable C14-immunoreactivity was observed predominantly in the cytoplasm of neurons throughout the brain. Neurons in the cerebral cortices showed moderate to intense immunoreactivity for $\mathrm{C} 14$ (Fig. 1A). In the cerebral neocortices, large pyramidal neurons in layers III and V showed more intense immunoreactivity than those in layers II or IV. In the hippocampal complex, pyramidal neurons in the CA13 regions and the hilus, and granule cells in the dentate gyrus, showed prominent immunoreactivity for C14 (Fig. 2A, B). Large and small neurons in the amygdala, lateral geniculate body (Fig. 1B) and neurons in the basal nucleus of Meynert (Fig. 1C) showed prominent immunoreactivity for C14. Moderate immunoreactivity was observed in the large and small neurons of the putamen, motor neurons in the brain stem, Purkinje cells of the cerebellum (Fig. 1E), and in the neurons of the inferior olivary nucleus and dentate nucleus. Although their nature remains unknown, small brown dot-like structures were scattered in some of the brain regions (Fig. 1A-D) 


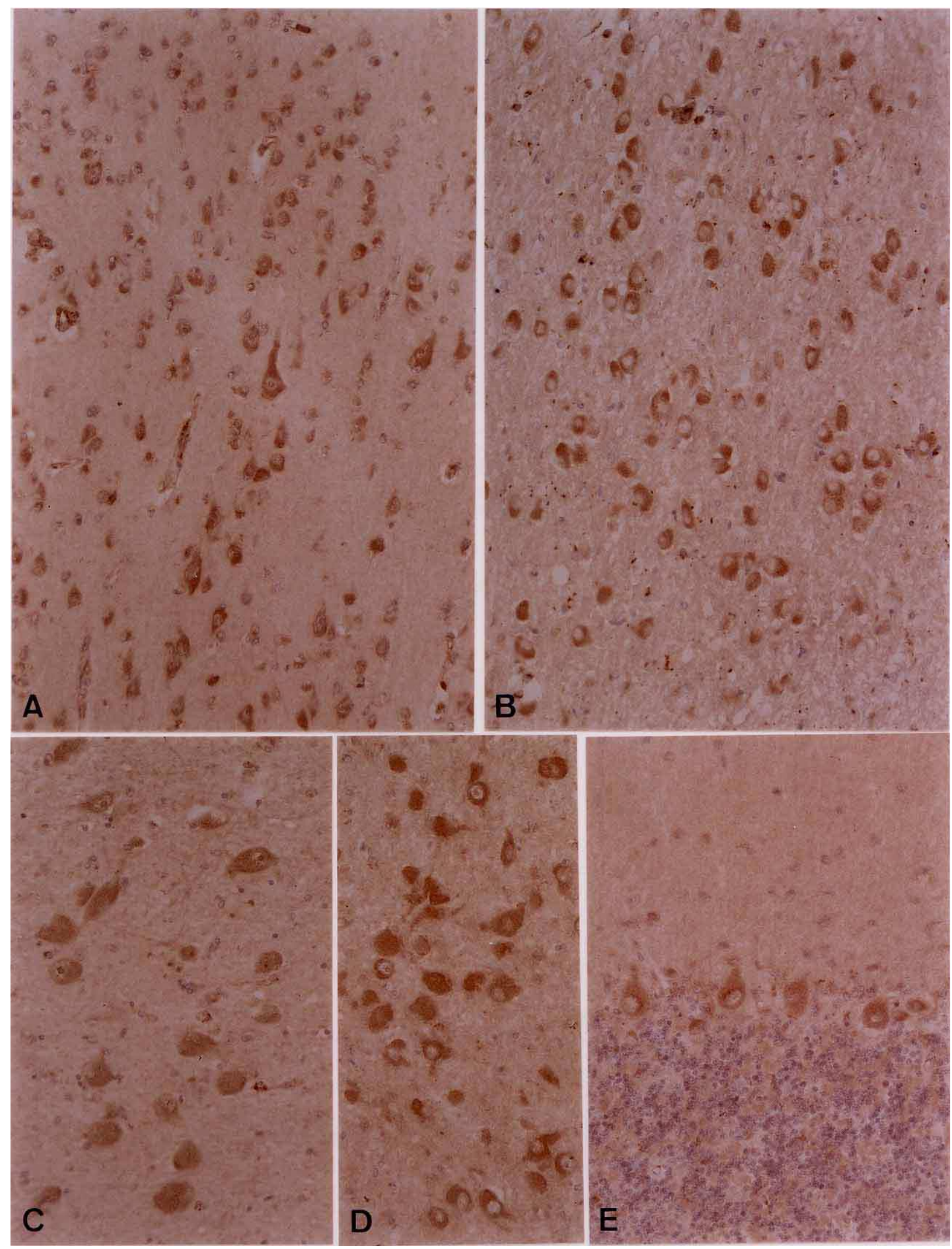

Fig. 1. Immunohistochemistry for anti-C14 antibody in various regions of control brains. Frontal cortex (A), lateral geniculate body (B), basal nucleus of Meynert $(\mathbf{C})$, posterior hypothalamic nucleus $(\mathbf{D})$ and cerebellum $(\mathbf{E})$, showing intense C14-immunoreactivity in the neuronal cytoplasm. Dots immunoreactive for $\mathrm{C} 14$ are scattered (A-D). $\times 50($ A-E). 

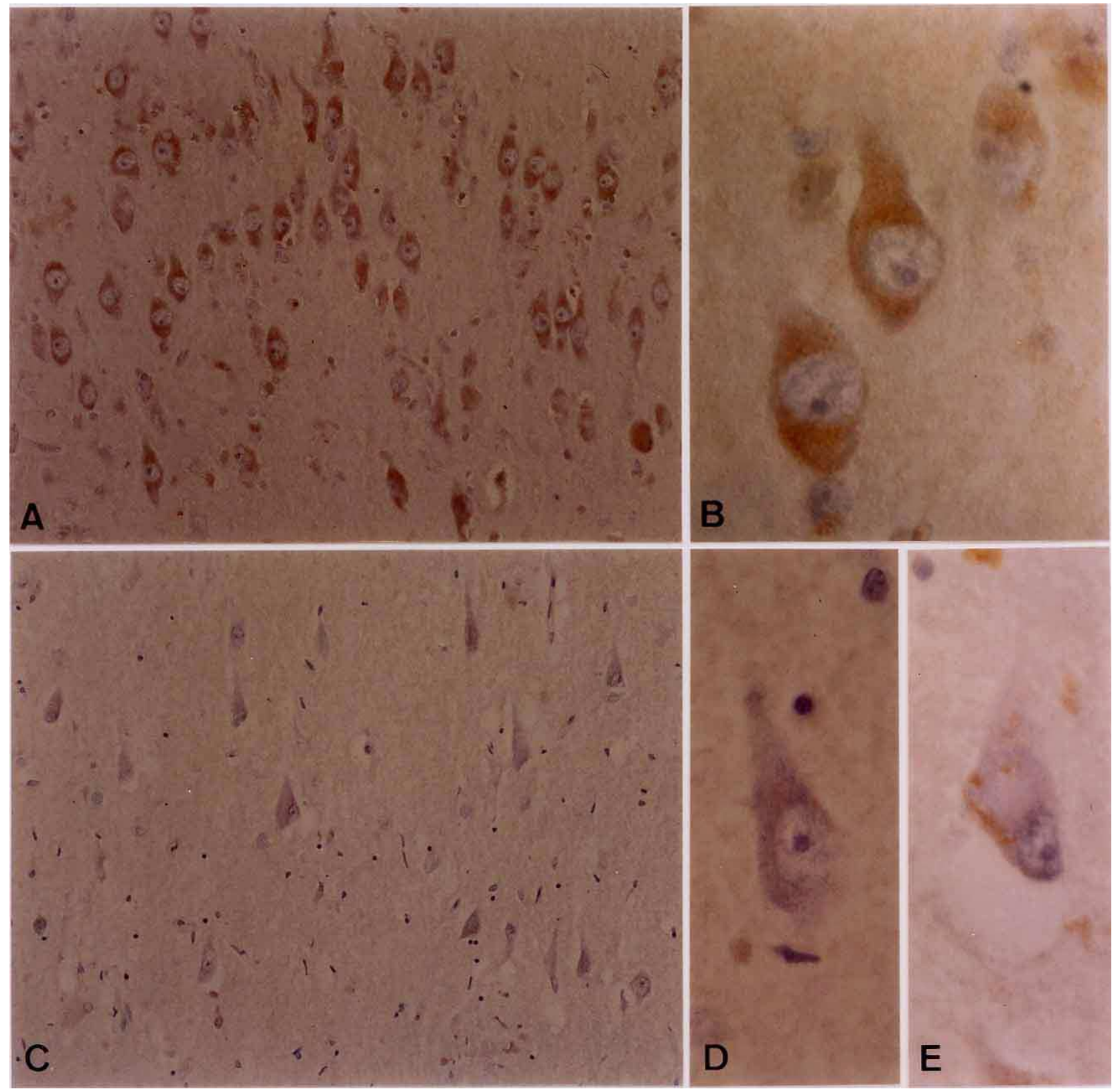

Fig. 2. Anti-C14 antibody immunohistochemistry in the CA1 region of the hippocampus. A, B: Control brains; pyramidal neurons, showing intense and diffuse C14-immunoreactivity in the cytoplasm. C-E: AD brains; pyramidal neurons showed markedly decreased C14-immunoreactivity accompanied by neuronal loss. Focal and weak immunoreactivity was observed in the neurons with neurofibrillary tangle $(\mathbf{E}) . \times 50(\mathbf{A}, \mathbf{C})$, $\times 200(\mathbf{B}, \mathbf{D}, \mathbf{E})$.

\section{Alzheimer's diseased brain (Fig. 2C-E, Fig. 3A-E)}

Neurons in the frontal, orbital, parietal, temporal and occipital cortices, basal ganglia, and thalamus showed slight immunoreactivity for C14. Throughout the neocortical layers the reactivity was reduced. Immunoreactivity for $\mathrm{C} 14$ was relatively spared in the lateral geniculate body, motor neurons in the brain stem, Purkinje cells of the cerebellum, and in the neurons of the inferior olivary nucleus and dentate nucleus. In the hippocampus and amygdala, many large neurons showed a marked reduction or loss of C14immunoreactivity (Fig. 2C). In particular, the C14-immuno- reactivity was virtually lost in the pyramidal neurons of the hippocampus (Fig. 2D). Neuritic plaques in the entorhinal cortex were stained intensely with the antibody for C14, whereas the plaques in other brain regions were stained to varying extents. Positively stained structures around or within the plaques suggest that plaque-associated microglia/ macrophages are immunoreactive for C14 (Fig. 3A, B). Neurons bearing neurofibrillary tangles tended to show a loss or a marked reduction in C14-immunoreactivity (Fig. 2E). Neurons with granulovacuolar degeneration did not show any C14-immunoreactivity in their cytoplasm or vacuoles. 

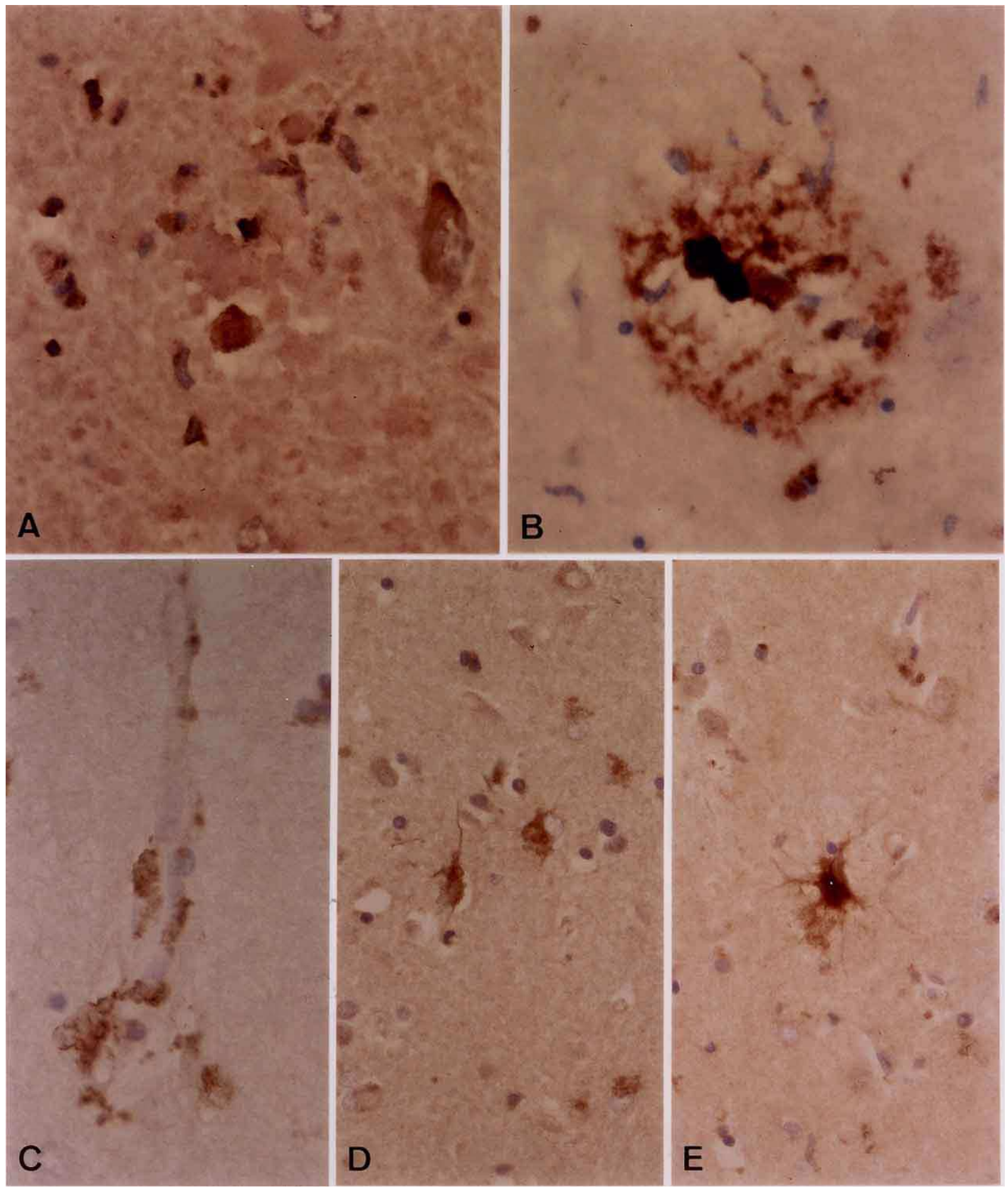

Fig. 3. Immunohistochemical stainings for anti-C14 antibody in $\mathrm{AD}$ brains. A, B: Entorhinal cortex. Dot-like C14-immunoreactivity in the neuritic plaques (A) as compared to A $\beta$-immunoreactive core in the same plaque (B). A: anti-C14 antibody. B: anti-A $\beta$ antibody (4G8). C: C14-immunoreactivity in perivascular microglia/macrophages in the thalamus. anti-C14 antibody. D, E: Astrocyte-like cells, showing C14immunoreactivity in the frontal cortex (D) and in the putamen (E). D, E: anti-C14 antibody. $\times 150(\mathbf{A}, \mathbf{B}), \times 100(\mathbf{C}, \mathbf{E}), \times 50$ (D).

Perivascular microglia/macrophages in the thalamus showed immunoreactivity for C14 (Fig. 3C). In the cerebral cortex and basal ganglia, some of the cells with morphological features of astrocytes were stained with antibody for C14 (Fig. 3D, E).

\section{Confocal laser microscopy (Fig. 4A-I, Fig. 5A-F)}

By using double-label laser confocal microscopy, we studied the hippocampi from the normal control as well as from the $\mathrm{AD}$ brains. Confocal microscopy allowed us to analyse colocalization of $\mathrm{HBCPB}$ (red) and other proteins 

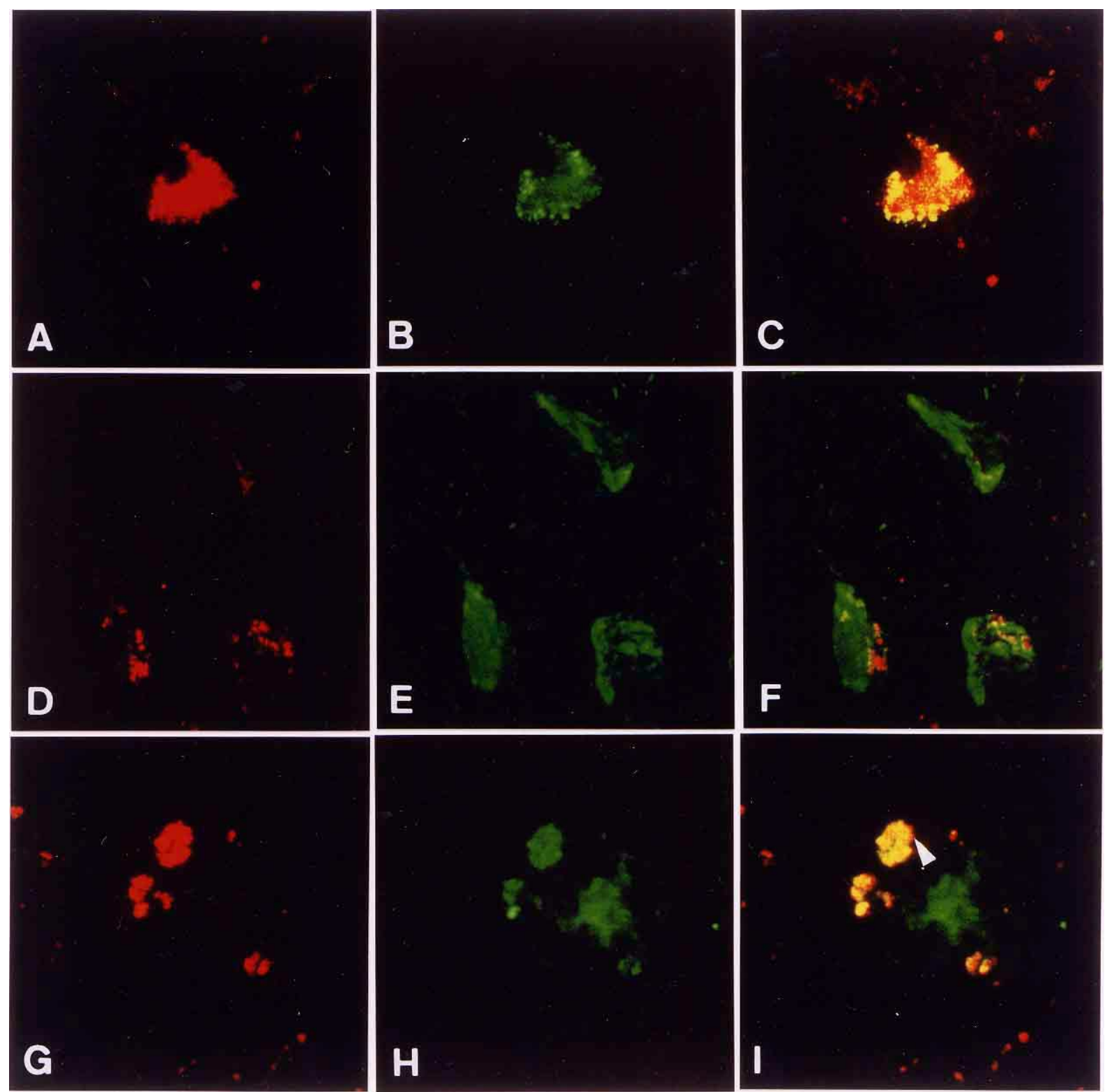

Fig. 4. Double-label laser confocal microscopy in AD brains. A-C, G, F, I: Double labeling with anti-C14 antibody (red) and anti-A $\beta$ antibody (4G8) (green). A-C: Pyramidal neurons in the CA1, showing granular colocalization (yellow) of C14- (red) and A $\beta$ - (green) immunoreactivity just beneath the cell membrane. D-F: Double labeling with anti-C14 antibody (red) and anti-PHF-tau antibody (AT8) (green). Neurofibrillary tangle bearing neurons lacked colocalization of PHF-tau and C14-immunoreactivity. G-I: Neuritic plaque, showing dot-like colocalization (yellow, arrow head) of C14- (red) with A $\beta$ - (green) immunoreactivity, surrounding the amyloid core. $\times 151$ (A-I).

including NF, A $\beta$, PHF-tau, GFAP and CD68 (green).

In the control brains, C14-immunoreactivity was distributed exclusively in neuronal cytoplasm showing colocalization with NF (data not shown). C14-immunoreactivity was found neither in axons nor in dendrites. In hippocampi from $\mathrm{AD}$ brains, a few of the spared pyramidal neurons showed colocalization of C14-(red) and A $\beta 17-24$-immunoreactivity (green) (Fig. 4A-C). In some of the hippocampal neurons bearing PHF-tau-positive neurofibrillary tangles, a patchy immunoreactive pattern for $\mathrm{C} 14$ was seen. However, colocalization of PHF-tau (green) and C14-immunoreactivity (red) was not evident (Fig. 4D-F). In some neuritic plaques, C14-immunoreactive structures (red) exhibited colocalization (yellow) with $\mathrm{A} \beta$ (green) (Fig. 4G-I) or CD68 (green) (Fig. 5A-C), a lysozomal marker for microglia/ macrophages. In the neuritic plaques, dystrophic neurites showed intense immunoreactivity for PHF-tau; however, there was no evidence of colocalization with PHF-tau and C14-immunoreactivity (data not shown). Large numbers of subpial astrocytes exhibited colocalization (yellow) of GFAP (green) with C14-immunoreactivity (red) (Fig. 5DF). 

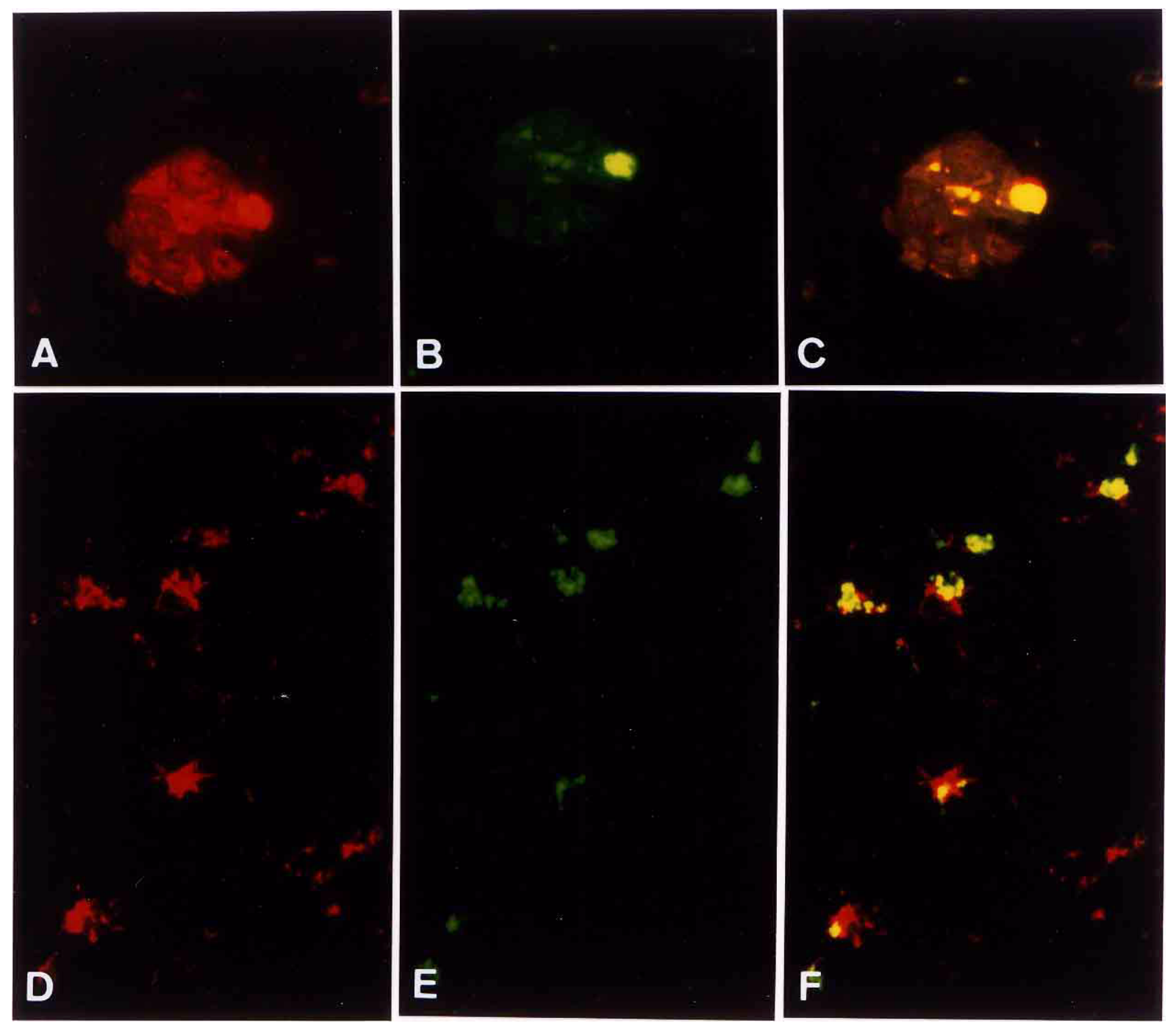

Fig. 5. Double-label laser confocal microscopy in AD brains. A-C: Double labeling of senile plaque with anti-C14 antibody (red) and anti-CD68 antibody (green), showing colocalization (yellow) with C14- and CD68-immunoreactivity. D-F: Double labeling of the molecular layer of the temporal cortex with anti-C14 antibody (red) and anti-GFAP antibody (green). GFAP-positive astrocytes showed colocalization (yellow) of C14-immunoreactivity. $\times 151(\mathbf{A}-\mathbf{F})$.

\section{Discussion}

Extracellular accumulation of $\mathrm{A} \beta$ and neuronal death in the cerebral cortices are hallmarks of Alzheimer's disease $[7,23]$. Intensive molecular genetical studies on the pedigrees with familial AD have revealed that mutations in the genes encoding the APP [5, 15], presenilin-1 and $-2[21,25]$ are responsible for the pathogenesis. In vitro $[8,11,22]$ as well as in vivo experiments using transgenic animals $[19,26]$ have corroborated "A $\beta$-cascade hypothesis" as a key underlying mechanism for AD pathogenesis. In this context, it is worth studying the regulatory mechanisms for APP processing by which $A \beta$ is produced. There are a wide variety of factors which are known to be involved in APP processing; neurotransmitters $[16,17]$, PKC-dependent and PKC-independent intracellular signaling pathways $[1,18]$, mitogenactivated protein kinase (MAPK) pathways [13], estrogen
[29], cholesterol [6, 20], apolipoprotein E [6] and stressactivated pathways [3].

Recently, Gervais et al. reported that caspases directly cleave APP during apoptosis, resulting in elevated A $\beta$ peptide formation [4]. The predominant site of caspasemediated proteolysis is within the cytoplasmic tail of APP, and cleavage at this site occurs in the hippocampal neurons in vivo following acute excitotoxic or ischemic brain injury. Vassar-R et al. identified $\beta$-site APP-cleaving enzyme (BACE), a transmembrane aspartic protease, which cleaves APP-derived substrates with the same sequence specificity as $\beta$-secretase [27].

We have recently isolated, from human hippocampus, an APP-processing protease (HBCPB) which cleaves native APP $[9,10]$. To gain a better insight into the functional role of HBCPB, we studied the immunohistochemical distribution of $\mathrm{HBCPB}$ in normal and $\mathrm{AD}$ brains by using the $\mathrm{C}$ - 
terminal specific antibody against $\mathrm{HBCPB}$, which can recognize solely a brain-specific carboxypeptidase B. In normal brains, we were able to demonstrate diffuse C14-immunoreactivity in the neuronal cytoplasm of the cerebral neocortices, hippocampi, amygdala, basal ganglia, thalamus, brain stem, cerebellar cortices and deep cerebellar nuclei. These observations suggest that HBCPB is a ubiquitously expressed protease that might be involved in the APP cleavage in neurons of the human brain. It is worth mentioning that C14-immunoreactivity was most prominent in hippocampi, amygdala, basal nucleus of Meynert, lateral geniculate body and large pyramidal neurons of the cerebral neocortex.

On the contrary, in AD brains, C14-immunoreactivity was decreased in the brain regions examined except for lateral geniculate body, brain stem and cerebellum. However, the extent of the reduction of C14-immunoreactivity was most severe in hippocampi and amygdala, which are highly susceptable to AD pathogenesis.

In other neurodegenerative diseases with no $A \beta$ deposition, including motor neurons in amyotrophic lateral sclerosis, substantia nigra and putamen in Parkinson's disease, putamen, pons, cerebellum and inferior olivary nucleus in multiple system atrophy, internal segment of globus pallidus, subtantia nigra, pons and cerebellum in MachadoJoseph disease, C14-immunoreactivity was preserved well in surviving neurons despite marked neuronal loss in affected brain regions (unpublished observation, Itoh K).

Taken together, it might be concluded that the reduced C14-immunoreactivity demonstrated in the present study is specific to $\mathrm{AD}$. If this were the case in sporadic $\mathrm{AD}$, reduction of HBCPB might be linked to impaired processing of APP followed by excessive $A \beta$ formation and deposition. It is tempting to speculate that $\mathrm{C} 14$-immunoreactive microglia/macrophages shown in senile plaques might be involved in the elimination of $A \beta$ deposition. In addition, it is noteworthy that $\mathrm{C} 14$-immunoreactive astrocytes were found in the outer layer of cerebral cortices and subcortical gray matter, but not in or around senile plaques. These astrocytes might be involved in compensatory regulation of APP processing under the situation where neuronal HBCPB activity was affected. Our observation that PHF-tau-positive neurons showed a loss or a marked reduction of C14immunoreactivity, favors the notion that HBCPB activity was suppressed in degenerating neurons.

HBCPB, a novel protease capable of cleaving APP, was ubiquitously expressed in neurons of the normal cerebral cortex, whereas the neuronal expression was reduced in AD brains. Further studies on the functions of HBCPB, using transgenic animals as well as in vitro systems would facilitate our understanding on the AD pathogenesis.

\section{Acknowledgements}

The authors greatly appreciate Dr. Kenji Ikeda, Dept. of Neuropathology, Tokyo Institute of Psychiatry, Tokyo Japan, for providing us valuable brain tissues with Alzheimer's disease.

\section{References}

1. Buxbaum, J. D., Ruefli, A. A., Parker, C. A., Cypess, A. A. and Greengard, P.: Calcium regulates processing of the Alzheimer amyloid protein precursor in a protein kinase $\mathrm{C}$-independent manner. Proc. Natl. Acad. Sci. U S A 91; 4489-4493, 1994.

2. Figueiredo-Pereira, M. E., Efthimiopoulos, S., Tezapsidis, N., Buku, A., Ghiso, J., Mehta, P. and Robakis, N. K.: Distinct secretases, a cysteine protease and a serine protease, generate the $\mathrm{C}$ termini of amyloid $\beta$-proteins $A \beta 1-40$ and $A \beta 1-42$, respectively. J. Neurochem. 72; 1417-1422, 1999.

3. Gabuzda, D., Busciglio, J., Chen, L. B., Matsudaira, P. and Yanker, B. A.: Inhibition of energy metabolism alters the processing of amyloid precursor protein and induces a potentially amyloidogenic derivative. J. Biol. Chem. 269; 13623-13628, 1994.

4. Gervais, F. G., Xu, D., Robertson, G. S., Vaillancourt, J. P., Zhu, Y., Huang, J., LeBlanc, A., Smith, D., Rigby, M., Shearman, M. S., Clarke, E. E., Zheng, H., Van Der Ploeg, L. H., Ruffolo, S. C., Thornberry, N. A., Xanthoudakis, S., Zamboni, R. J., Roy, S. and Nicholson, D. W.: Involvement of caspase in proteolytic cleavage of Alzheimer's amyloid- $\beta$ precursor protein and amyloidogenic A $\beta$ peptide formation. Cell 97; 395-406, 1999.

5. Goate, A., Chartier-Harlin, M. C., Mullan, M., Brown, J., Crawford, F., Fidani, L., Giuffra, L., Haynes, A., Irving, N. and James, L.: Segregation of missense mutation in the amyloid precursor protein gene with familial Alzheimer's disease. Nature 349; 704706, 1991.

6. Howland, D. S., Trusko, S. P., Savage, M. J., Reaume, A. G., Lang, D. M., Hirsch, J. D., Maeda, N., Siman, R., Greenberg, B. D., Scott, R. W. and Flood, D. G.: Modulation of secreted $\beta$ amyloid precursor protein and amyloid $\beta$-peptide in brain by cholesterol. J. Biol. Chem. 273; 16576-16582, 1998.

7. Iwatsubo, T.: Amyloid $\beta$ peptides and presenilins in the pathogenesis of Alzheimer's disease. Acta Histochem. Cytochem. 32; 13-15, 1999

8. Lambert, M. P., Barlow, A. K., Chromy, B. A., Edwards, C., Freed, R., Liosatos, M., Morgan, T. E., Rozovsky, I., Trommer, B., Viola, K. L., Wals, P., Zhang, C., Finch, C. E., Krafft, G. A. and Klein, W. L.: Diffusible nonfibrillar ligands derived from A $1-42$ are potent central nervous system neurotoxins. Proc. Natl. Acad. Sci. U S A 95; 6448-6453, 1998.

9. Matsumoto, A., Itoh, K. and Matsumoto, R.: A novel carboxypeptidase $\mathrm{B}$ that processes native $\beta$-amyloid precursor protein is present in human hippocampus. Eur. J. Neurosci. 12; 227-238, 2000.

10. Matsumoto, A., Matsumoto, R., Enomoto, T. and Itoh, K.: A human brain proteolytic activity capable of cleaving natural $\beta$ amyloid precursor protein is affected by its substrate glycoconjugates. Neurosci. Lett. 242; 109-113, 1998.

11. Mattson, M. P.: Cellular actions of $\beta$-amyloid precursor protein and its soluble and fibrillogenic derivatives. Physiol. Rev. 77; 1081-1132, 1997.

12. Mills, J. and Reiner, P. B.: Regulation of amyloid precursor protein cleavage. J. Neurochem. 72; 443-460, 1999.

13. Mills, J., Laurent Charest, D., Lam, F., Beyreuther, K., Ida, N., Pelech, S. L. and Reiner, P. B.: Regulation of amyloid precursor protein catabolism involves the mitogen-activated protein kinase signal transduction pathway. J. Neurosci. 17; 9415-9422, 1997.

14. Mirra, S. S., Heyman, A., McKeel, D., Sumi, S. M., Crain, B. J., Brownlee, L. M., Vogel, F. S., Hughes, J. P., van Belle, G. and Berg, L.: The consortium to establish a registry for Alzheimer's disease (CERAD) Part II. Standardization of the neuropathologic assessment of Alzheimer's disease. Neurology 41; 479-486, 1991.

15. Mullan, M., Crawford, F., Axelman, K., Houlden, H., Lilius, L., 
Winblad, B. and Lannfelt, L.: A pathogenic mutation for probable Alzheimer's disease in the APP gene at the N-terminus of $\beta$ amyloid. Nat. Genet. 1; 345-347, 1992.

16. Nitsch, R. M., Slack, B. E., Wurtman, R. J. and Growdon, J. H.: Release of Alzheimer amyloid precursor derivatives stimulated by activation of muscarinic acetylcholine receptors. Science 258; 304-307, 1992.

17. Nitsch, R. M., Deng, A., Wurtman, R. J. and Growdon, J. H.: Metabotropic glutamate receptor subtype mGluRa stimulate amyloid $\beta$-protein precursor ectodomain. J. Neurochem. 69; 704712, 1997.

18. Nitsch, R. M., Slack, B. E., Farber, S. A., Schulz, J. G., Deng, M., Kim, C., Borghesani, P. R., Korver, W., Wurtman, R. J. and Growdon, J. H.: Regulation of proteolytic processing of the amyloid beta-protein precursor of Alzheimer's disease in transfected cell lines and in brain slices. J. Neural. Transm. (Suppl.) 44; $21-$ 27, 1994.

19. Price, D. L., Wong, P. C., Markowska, A. L., Lee, M. K., Thinakaren, G., Cleveland, D. W., Sisodia, S. S. and Borchelt, D. R.: The value of transgenic models for the study of neurodegenerative diseases. Ann. N Y Acad. Sci. 920; 179-191, 2000.

20. Racchi, M., Baetta, R., Salvietti, N., Ianna, P., Franceschini, G., Paoletti, R., Fumagalli, R., Govoni, S., Trabucchi, M. and Soma, M.: Secretary processing of amyloid precursor protein is inhibited by increase in cellular cholesterol content. J. Biochem. 322; 893898, 1997.

21. Rogaev, E. I., Sherrington, R., Rogaeva, E. A., Levesque, G., Ikeda, M., Liang, Y., Chi, H., Lin, C., Holman, K. and Tsuda, T.: Familial Alzheimer's disease in kindreds with missense mutations in a gene on chromosome 1 related to the Alzheimer's disease type 3 gene. Nature 376; 775-778, 1995.

22. Roher, A. E., Chaney, M. O., Kuo, Y. M., Webster, S. D., Stine, W. B., Haverkamp, L. J., Woods, A. S., Cotter, R. J., Tuohy, J.
M., Krafft, G. A., Bonnell, B. S. and Emmerling, M. R.: Morphology and toxicity of $A \beta-(1-42)$ dimer derived from neuritic and vascular amyloid deposits of Alzheimer's disease. J. Biol. Chem. 271; 20631-20635, 1996.

23. Selkoe, D. J.: The molecular pathology of Alzheimer's disease. Neuron 6; 487-498, 1991.

24. Selkoe, D. J.: Cellular and molecular biology of $\beta$-amyloid precursor and Alzheimer's disease. In "The Molecular and Genetic Basis of Neurological Disease", ed. by S. B. Prusiner, R. N. Rosenberg, S. D. Mauro, et al., Butterworth Heinemann Press, Boston, 1997, pp. 601-602.

25. Sherrington, R., Rogaev, E. I., Liang, Y., Rogaeva, E. A., Levesque, G., Ikeda, M., Chi, H., Lin, C., Li, G. and Holman, K.: Cloning of a gene bearing missense mutations in early-onset familial Alzheimer's disease. Nature 375; 754-760, 1995.

26. Turner, R. S.: Alzheimer's disease in man and transgenic mice: females at higher risk. Am. J. Pathol. 158; 797-801, 2001.

27. Vassar, R., Bennett, B. D., Babu-Khan, S., Kahn, S., Mendiaz, E. A., Denis, P., Teplow, D. B., Ross, S., Amarante, P., Loeloff, R., Luo, Y., Fisher, S., Fuller, J., Edenson, S., Lile, J., Jarosinski, M. A., Biere, A. L., Curran, E., Burgess, T., Louis, J. C., Collins, F., Treanor, J., Rogers, G. and Citron, M.: Beta-secretase cleavage of Alzheimer's amyloid precursor protein by the transmembrane aspartic protease BACE. Science 286; 735-741, 1999.

28. Wilson, C. A., Doms, R. W. and Lee, V. M-Y.: Intracellular APP processing and A $\beta$ production in Alzheimer's disease. J. Neuropathol. Exp. Neurol. 58; 787-794, 1999.

29. Xu, H., Gouras, G. K., Greenfield, J. P., Vincent, B., Naslund, J., Mazzarelli, L., Fried, G., Jovanovic, J. N., Seeger, M., Relkin, N. R., Liao, F., Checler, F., Buxbaum, J. D., Chait, B. T., Thinakaran, G., Sisodia, S. S., Wang, R., Greengard, P. and Gandy, S.: Estrogen reduces neuronal generation of Alzheimer $\beta$-amyloid peptides. Nat. Med. 4; 447-451, 1998. 